\section{Aspectos éticos en la participación de personas con diversidad funcional en proyectos de investigación tecnológica ${ }^{1}$}

\section{Ethical issues in the participation of people with functional diversity in technological research projects}

\section{Resumen}

La involucración de personas con diversidad funcional en proyectos de investigación tecnológica tiene ciertas peculiaridades en el plano ético que deben ser consideradas por todos aquellos implicados en el proceso. El estudio de estas consideraciones desde un punto de vista teórico sustentado por la praxis nos lleva a presentar una aproximación a los problemas de orden práctico que plantean estas actividades. Ante la carencia de protocolos establecidos, presentamos en este artículo un marco integrado de evaluación ética que considere como parámetros principales a tener en cuenta el trato adecuado, el respeto a la privacidad, el estímulo a la expresión de opiniones, la gestión de las relaciones personales establecidas y la decepción por parte de los sujetos participantes.

\section{Palabras clave}

Ética, investigación, participante, privacidad, comportamiento, protocolo, involucración.

I. Este artículo se inscribe en el proyecto de investigación APSIS 4 all y Cloud 4 All: Cloud Platforms Lead to Open and Universal Acess for People with Disabilities and for All, financiado por la Unión Europea (EU FP7), en el que ambos autores participan como miembros del E\&LAC (Ethical and Legal Advisory Committee) y en el proyecto de investigación "Ciencia, tecnología y sociedad: problemas políticos y éticos de la computación en nube como nuevo paradigma sociotecnológico” (FFI20I3-46908-R).

\begin{abstract}
The participation of people with disabilities in technological research projects has certain peculiarities on an ethical level that should be considered by all those involved in the process. The study of these considerations from a theoretical point of view, also supported by praxis, lead us to present an approach to the practical problems posed by these activities. In the absence of established protocols, this paper presents an integrated ethical review framework that considers following parameters as fundamental proper treatment, respect for privacy, stimulus to express opinions, correct management of the personal relationships and the disappointment of the participating persons.
\end{abstract}

\section{Keywords}

Ethics, research, participant, privacy, behaviour, protocol, involvement.
Fausto Sainz de Salces

<faustosainzsalces@gmail.com>

Technosite

\section{Javier Bustamante Donas}

Prof. Titular Departamento de Filosofía del Derecho, Moral y Política II. Universidad Complutense de Madrid

Para citar:

Sainz de Salces, F. y Bustamante Donas, J. (20I4): “Aspectos éticos en la participación de personas con diversidad funcional en proyectos de investigación tecnológica”. Revista Española de Discapacidad, 2 (I): I65-I74.

<http://dx.doi.org/I0.5569/23405104.02.01.09>

Fecha de recepción: 24-09-20I3 Fecha de aceptación: I9-05-20I4 


\section{Introducción}

Avanzar en el campo de la tecnología, y especialmente en la tecnología asistencial, implica necesariamente la involucración de personas con diversidad funcional o aquellas con necesidades especiales en los procesos de diseño y evaluación de los productos que se quieren desarrollar. Idealmente no debería tratarse tan solo de involucrar a las personas de este colectivo -que además van a ser, en muchos casos, los principales destinatarios de la tecnología- en los procesos de diseño y evaluación, sino durante todo el proceso de desarrollo: desde la misma concepción de la idea hasta su finalización. Y, en algunos casos, hasta más allá de su lanzamiento, prestando especial atención a los usos innovadores sugeridos a través de su adopción por parte de los usuarios finales. En este sentido la participación de personas con diversidad funcional puede plantearse también como un derecho pues, si bien los fundamentos económicos y pertinentes a la competitividad se presentan como obvios, quizás un avance en el terreno legal favorecería la involucración de este colectivo en los desarrollos tecnológicos de forma amplia.

Además, se hace necesario redefinir algunos de los parámetros con los que se han caracterizado tradicionalmente a los usuarios de estas tecnologías. Adoptamos el término "diversidad funcional" frente a expresiones como "discapacitados o portadores de necesidades espaciales”. Defendemos que ambas expresiones se han revelado históricamente como instrumentos de construcción social que descaracterizan a las personas, al destacar apenas los factores limitadores, disfuncionales y creadores de dependencia, elevándolos a una categoría ontológica. Promueven, además, formas de ver su realidad que nos aleja a todos de una visión reveladora de la auténtica naturaleza de estas personas y de la metodología de innovación y desarrollo más digna y conveniente para lidiar con los problemas de diseño tecnológico orientado a estos colectivos. Dentro del marco general de desarrollo de los paradigmas de diseño vemos cómo el diseño participativo ha cobrado especial vigor en las dos últimas décadas, y cómo esta inclusión de los posibles usuarios en los procesos de desarrollo se ha ido extendiendo a casi todos los campos de la tecnología. Existen, empero, dificultades a la hora de desarrollar plenamente y con integridad ética aquellas prácticas y actividades demandadas por el diseño participativo, que tanto beneficio acarrean a la comunidad con diversidad funcional. Asuntos relacionados con la privacidad, la comunicación, el trato adecuado, o la decepción, vendrían a conformar un cuerpo de asuntos que deben ser considerados desde un punto de vista deontológico al trabajar con personas portadoras de necesidades especiales en entornos de investigación.

Asimismo, debe tenerse en cuenta que el análisis de todas estas cuestiones que se presentan aquí tiene como trasfondo la praxis, fundamentada en la participación en proyectos de investigación de carácter nacional e internacional.

Los dilemas de carácter ético son inherentemente complejos, pueden ser difíciles de reconocer y podrían tener en muchos casos implicaciones ambiguas (Thiel et al., 20I2). A pesar de que las preocupaciones éticas se reflejan indirectamente en los códigos de conducta de las organizaciones profesionales, a las que algunos de los investigadores pertenecen, todavía es necesario incluirlas explícitamente en los planes estratégicos durante la primera fase de los proyectos de investigación, sobre todo en aquellos que hacen del diseño participativo su paradigma.

La transmisión de los principios éticos a la práctica es, metafóricamente hablando, un campo de minas (Swain et al., I998). Los principios éticos generales pueden verse amenazados por las propias características de diseño de los proyectos de investigación. En este sentido, tratar de desarrollar un conjunto de directrices éticas no tiene porqué adaptarse a las necesidades de cada investigación en particular. Sin embargo, se espera que al hacerlo al menos puedan evitarse daños, o incluso eliminarlos, si la causa es un conocimiento insuficiente de 
estos aspectos éticos por parte de personas con un perfil más técnico: los desarrolladores. La preparación de directrices y el suministro de cualquier otra información, como la incluida en el formulario de consentimiento informado, puede servir al propósito de asegurar la concienciación acerca de la responsabilidad ética por parte de los desarrolladores, dado su papel estratégico en los proyectos, como propone Gram-Hansen (2009).

El entorno de diseño participativo se presta a lo que Turilli (2007) ha llamado "el problema de la consistencia ética”, en el que es difícil fiscalizar a los diferentes actores inmersos en el proceso para asegurar que los resultados, en términos de productos, servicios o metodologías, son éticamente coherentes. Otro factor a tener en cuenta es la importancia del conocimiento informal, el basado en la experiencia y los conocimientos -que pueden entrar en juego cuando sea necesario- provenientes de diferentes fuentes. Tenemos que asegurarnos de que las personas con diversidad funcional puedan comunicarse de manera clara y eficiente, sin perjudicar su dignidad y sin provocarles ningún tipo de incomodidad. En particular nos referimos a personas con diversidad funcional cognitiva más que sensorial o motora. Es en este colectivo en el que los problemas relacionados con su conciencia, autonomía, consentimiento informado y gestión de la decepción cobran un valor especial que requiere un tratamiento ético más delicado y profundo. Comprender las tecnologías innovadoras y sus posibles usos, así como la reciprocidad entre dichas tecnologías y los seres humanos, es esencial para aprovechar cualquier diseño futuro.

Otro factor que puede afectar el proceso de toma de decisiones tiene que ver con la consciencia del participante-voluntario acerca de las consecuencias (incluidas las éticas) de un diseño, siempre específico, desarrollado en el proceso de innovación. Como demuestra Manders-Huits (20I0), cualquier diseño tiene implicaciones éticas, y sabiendo que la tecnología no es neutral al estar cargada de valores, es conveniente disponer de medios para evaluar y justificar éticamente las decisiones tomadas durante el diseño de la tecnología. No solo estamos diseñando simplemente herramientas eficaces (que maximicen la utilidad esperada de las consecuencias de las decisiones) o eficientes (que maximicen la optimización de los recursos en la consecución del objetivo propuesto). Deben ser herramientas buenas, es decir, acordes con los parámetros éticos que se establezcan. Por lo tanto, los participantes deben ser lo más conscientes posible de las actividades de diseño. $\mathrm{Al}$ ser usuarios de dichas herramientas, tienen un interés legítimo en el proceso de desarrollo de las mismas. Su participación en todas las etapas del desarrollo ayudaría a aumentar los aspectos beneficiosos y diagnosticar y minimizar los efectos colaterales no deseables.

\section{Trato adecuado como base de las relaciones entre investigadores y voluntarios}

Es importante comprobar que todo el personal de investigación que trata directamente con los voluntarios (entrevistadores, conductores de prueba, facilitadores, etc.) ha recibido una formación adecuada. En primer lugar, a fin de poder identificar a las personas que carecen de la capacidad de dar su consentimiento, y de esta manera poder encontrar la forma de obtener ese consentimiento tan necesario. En este caso el consentimiento ha de obtenerse de alguien cercano a la persona interesada que puede aconsejarle sobre si él o ella quiere estar involucrado. Pero aquí nos encontramos de nuevo con un problema de certeza. Como bien dice Stalker (I 998), en el caso de las personas con dificultades de aprendizaje o con diversidad funcional cognitiva, ¿cómo sabemos si esta persona realmente entiende lo que le estamos pidiendo? ¿Cómo sabemos si realmente quieren participar en las pruebas?

También tenemos que considerar las implicaciones que la experiencia del proceso de investigación y desarrollo puede tener en el investigador, y los riesgos que puede representar 
el desviarse de la agenda de investigación al involucrarse demasiado en las vidas de los participantes (Stalker, I998). Este es un caso bien conocido en los estudios antropológicos, donde los procesos simpáticos entre el investigador y los participantes pueden viciar los resultados. Frente a ello, es necesario un cierto extrañamiento por parte del investigador que permita una observación más objetiva y menos emocional. Y, por otro lado, esta consideración acerca de la experiencia es esencial para poder relacionarse con las personas con diversidad funcional de una manera apropiada. En ese sentido, además de evitar la estigmatización de los participantes es importante hacer uso de un protocolo ético aceptable (Vines et al., 20I3).

En este sentido, la noción de trato adecuado va mucho más allá de la típica definición de responsabilidad por el sujeto humano basada en la evitación del daño al sujeto (avoiding harm), ya que no hay una única responsabilidad que recaiga de forma unitaria sobre una persona médicamente cualificada. Los problemas que aborda el proyecto PERSONA² no son problemas médicos, sino de un rango mucho mayor de acción. Por tanto, las soluciones no son solo médicas. La evitación del daño al sujeto debe obedecer a parámetros más globales. (Ejemplo: si un aparato de medida ofrece una lectura errónea, ¿quién es responsable por un diagnóstico o una acción basada en dicha medida?). Tratamos con sistemas que pueden ser de alta o baja complejidad, pero a menudo de un alto grado de acoplamiento (high coupling). Esto significa que si un sensor ofrece información errónea, todas las decisiones que se tomen después estarán contaminadas. Por ello habrá que definir la responsabilidad de cada uno de los actores de manera relacional con los demás. Cada uno tiene una parcela de responsabilidad definida e identificada, pero debe actuar con visión periférica, siendo consciente de cómo los parámetros de su actividad influyen sobre las actividades a cargo de los demás responsables. Desde el punto de vista legal no se hace evidente el problema, ya que las responsabilidades de cada uno se

2. <http://www.aal-persona.org/> delimitan por contrato. Desde el punto de vista ético, sin embargo, es recomendable profundizar en esta noción de trato adecuado desde una perspectiva relacional. Por todo ello, el trato adecuado que debe observarse en el contexto de la investigación implica además relaciones alentadas por las virtudes, y no solamente relaciones basadas en el principio de no malevolencia y de responsabilidad. En este sentido, hay que dar un paso más allá para introducir criterios axiológicos que enfaticen el bienestar de los sujetos participantes a través tanto de las diferentes formas que cada colectivo tenga de entender su propio bien, como a partir de procedimientos que garanticen dos formas de trato adecuado basado en el principio de beneficencia: I) un trato igualitario basado en la asignación del mayor rango posible de derechos, que sea compatible con la misma asignación al resto de los participantes, 2) y un trato preferente que aplique un principio de beneficencia de geometría variable, siempre que tales diferencias redunden en un beneficio particular para los más desfavorecidos y no supongan perjuicios al resto y sean de público conocimiento antes del comienzo de la investigación. En definitiva, a la excelencia científica de la investigación debe unirse su relevancia ética y social.

\section{Respeto al derecho a la libre expresión por parte del participante}

Las actividades de co-diseño, las sesiones de grupo y otras técnicas, son herramientas de colaboración muy populares en ciertos ambientes de diseño participativo para asegurarse de que todos los interesados tienen una oportunidad de expresar sus puntos de vista y sus ideas. Incluso cuando se compruebe que todos los usuarios potenciales -directos e indirectos- se integran en el proceso y la evaluación de la tecnología que está en marcha existe otra cuestión que hay que contemplar: la cuestión de la honestidad que se deriva de varios factores. ¿Qué pasa si ciertos grupos de interés presionan para imponer sus puntos de vista en 
la sesión de diseño o evaluación (o proceso), incluso cuando sus opiniones son opuestas a las de los principales beneficiarios de la tecnología? ¿Cómo gestionar la presión de grupo que se puede generar en estas sesiones? En este sentido, es muy importante que los moderadores sean conscientes de estos riesgos y guíen las sesiones de tal manera que se eviten estos riesgos.

También será necesario introducir pautas en el protocolo de investigación que disminuyan el estrés de los participantes. Pequeños detalles pueden tener una gran influencia en su bienestar. Por ejemplo, el uso de dispositivos detectores de objetos sensibles para la calidad de vida del sujeto (llaves, cartera, etc.), que identifiquen su posición respondiendo con una señal ante un silbido o movimiento corporal discreto (aplauso, comando de voz, etc.). Las personas con leve demencia senil comienzan a esconder dichos objetos y después no recuerdan dónde se encuentran, con el consiguiente estrés. Estos aspectos instrumentales de la investigación cobran un papel más relevante por la naturaleza de los participantes y el respeto por su dignidad y sensibilidad.

\section{Gestión de la decepción: Retroalimentación y expectativas}

Una cuestión importante a tener en cuenta es la percepción de los resultados directos e indirectos de la investigación por parte de los participantes. En este sentido, aspectos como la decepción o la gestión de las expectativas (Stalker, I998) cobran especial relevancia. Por otra parte, también es cierto que la mayoría de los participantes no suelen preguntar por los resultados de las actividades realizadas; y cuando lo hacen, tienden a concentrar sus pesquisas en los resultados prácticos y las implicaciones directas para su vida. Parece prevalecer con frecuencia el interés pragmático sobre el científico.
Otro tema importante, cuando se presenta a usuarios tecnología que podría ser de gran ayuda para ellos, es la cuestión de la decepción. La decepción puede aparecer en diferentes formas durante la investigación. En especial, en personas con diversidad funcional y/o de edad avanzada. Por ejemplo, los participantes en los estudios pueden decepcionarse, e incluso sentirse de algún modo engañados, cuando termina el estudio y se les retira la tecnología con la cual han estado interactuando (Barcenilla y Tijus, 20I2), especialmente si dicha tecnología hace su vida diaria más cómoda y fácil. En el caso de las personas con diversidad funcional cognitiva, la cuestión de la decepción también se puede examinar desde otro punto de vista: el desarrollo de vínculos afectivos o vínculos con los miembros del proyecto, miembros del equipo de investigación y compañeros participantes, como hemos señalado anteriormente. Esto también le puede suceder a la población en general, pero en el caso de la participación de personas con este perfil se produce con una frecuencia que hace de este fenómeno un factor que no es periférico ni anecdótico (Swain et al., I998). Así, es fácil que se produzcan situaciones caracterizadas por un fuerte apego emocional.

Es por ello que tanto los desarrolladores como los demás agentes involucrados en la investigación deben considerar cuidadosamente la experiencia actual y las expectativas de futuro para cada uno de los grupos implicados. Para ello deben tener en cuenta las implicaciones para las relaciones a partir del horizonte de interpretación que cada uno de ellos proyecta en función de su experiencia personal y su posición en el proceso de innovación y desarrollo.

En los proyectos en los que hemos participado hemos pensado en la manera de tratar una posible decepción por parte de los participantes y de evitar una percepción de expectativas insatisfechas, a pesar de que no todos los proyectos en los que hemos estado involucrados necesitaban de constante interacción con tecnología durante largos periodos de tiempo (APSIS, CLOUD4all). La solución adoptada consistió en utilizar el consentimiento informado como una primera declaración de intenciones 
clara para evitar la decepción que pudiese generarse en los experimentos o interacciones de la tecnología y los voluntarios -especialmente en aquellos que interactuaban con la tecnología una sola vez- y también para recordar a los participantes que la tecnología estudiada estaba en proceso de desarrollo. A los participantes también se les recordó que si querían se les informaría sobre el progreso en la investigación.

Sin embargo, antes de todas estas acciones para llevar a cabo la investigación con personas que carecen de la capacidad necesaria para autorizar su propia participación en las actividades, es necesario obtener la aprobación de un órgano apropiado, tal como un Comité de Ética Local o un Comité de Ética de la Investigación. El proceso de aprobación puede ser muy lento y esto debe ser tenido en cuenta, tomando las medidas necesarias para obtener la aprobación desde el comienzo del proyecto.

\section{Uso responsable del consentimiento informado}

Uno de los principales problemas éticos durante el desarrollo de la metodología de la investigación en los proyectos APSIS y CLOUD 4all estaba relacionado con la necesidad de considerar si la investigación podía involucrar a personas carentes de la plena capacidad para tomar sus propias decisiones, lo que incidía claramente sobre la aplicación del consentimiento informado. Esto podría incluir a personas con casos concretos de diversidad funcional que se manifieste en escenarios con déficits profundos de aprendizaje, o que presenten ciertos deterioros cognitivos graves o de comunicación, que pueden no ser capaces de dar su consentimiento informado para participar en el estudio. Una de las principales cuestiones relativas a la inclusión de participantes con diversidades funcionales cognitivas es el formulario de consentimiento. A pesar de que en la mayoría de los casos la persona responsable del sujeto propuesto es quien acepta o rechaza la participación de éste en el proyecto, siempre es una muestra respetuosa preparar un formulario de consentimiento que sea fácil de entender. Los problemas pueden surgir cuando hay una discrepancia entre los posibles participantes y la opinión de la persona legalmente responsable sobre la voluntad plena para participar en la investigación.

Durante las diferentes pruebas llevadas a cabo en el marco del proyecto APSIS, las observaciones hechas por los participantes, en su mayoría con deterioro cognitivo, abundan sobre la necesidad de mantener simple el estilo del consentimiento informado. Esto refleja la regulación actual (Nys, 20I2) y en ese sentido podría ser aconsejable tener varias versiones del consentimiento informado, a fin de poder presentar a cada participante aquella que le resulte más fácil de entender de acuerdo a la capacidad cognitiva de los interesados.

En relación con la gestión adecuada de la decepción, el consentimiento informado ayuda técnicamente a presentar a los participantes las posibilidades y realidades posteriores al periodo de participación en relación con la tecnología estudiada. No obstante, su alcance es limitado; el uso del sentido común y un acercamiento personalizado que prepare a los participantes para el fin de la experiencia y sus consecuencias parece necesario y adecuado.

\section{La metáfora de la diáspora en los casos de deterioro cognitivo}

Algunos riesgos que plantea la utilización de tecnologías por parte de personas con diversidad funcional tiene que ver con factores de edad y con casos concretos de déficits cognitivos. En estos casos particulares, el empleo de este tipo de personas requiere una serie de precauciones especiales, pues existe la tentación de no utilizar este perfil como sujeto experimental, o de hacerlo empleando rígidos protocolos que acaban anulando su autonomía. En estos casos 
particulares que requieren un uso particular de los principios de beneficencia y responsabilidad, pueden diseñarse modelos de trato adecuado considerando a estas personas como miembros con mayor o menor grado de integración en sus respectivas redes sociales. De forma genérica, la persona que sufre un daño cognitivo severo o un deterioro cognitivo relacionado con la edad vive en una especie de realidad diaspórica. La diáspora social consiste en estar apartado de tus raíces, de tus seres queridos, de tus costumbres, de tu tierra. El mundo es también un lugar extraño para la persona que presenta este escenario de deterioro cognitivo. Imaginemos una persona de avanzada edad con déficit cognitivo debido a una demencia senil leve o un Alzheimer incipiente. Sus condiciones físicas y mentales le apartan de sus costumbres, de la forma tradicional de realizar incluso las tareas más básicas. Todo lo que antes era cotidiano ahora queda más lejos, más inaccesible que lo que nunca había estado. Por eso vive un extrañamiento, una alienación con respecto a su mundo. Como principio metodológico, y como garantía para preservar el mayor grado de autonomía posible, estas tecnologías deberían aplicarse según este criterio de diáspora digital, en una escala establecida en relación al grado de distanciamiento de la persona con respecto de su realidad cotidiana.

Esta escala incide en el problema de la definición del usuario, ya que las personas que pueden beneficiarse de los resultados del proyecto no obedecen a un perfil unificado, sino a un abanico de condiciones mentales, dentro de una escala de autonomía personal. La forma de tratar con ese abanico es el concepto de inteligencia central frente a la inteligencia ambiental. Cuanto menos autónoma sea la persona, más inteligencia (capacidad de decisión, carga de información) estará situada en los aparatos que la ayudan. Entre ambas debe haber un equilibrio en función del estado de la persona y de su grado y especificidad de diversidad funcional. Las herramientas tecnológicas que aporten esta inteligencia ambiental deber ser lo menos invasivas posible.

Dado que las soluciones tecnológicas tendrán que adaptarse a un amplio espectro de sujetos en función de esta escala diaspórica, sería interesante también que, en la medida de lo posible, que las soluciones se que presenten al usuario sean modulares -en lugar de conformar un paquete cerrado-, de forma que pueda elegir aquellas con las que se sienta cómodo. (Ejemplo: la utilización de un dispositivo GPS para localizar a una persona. Puede ser útil para un usuario con demencia senil ligera, pero puede ser una intromisión a la intimidad de un usuario con plenas facultades mentales, que no precisa estar localizable por su familia). El consentimiento informado debe tener siempre en cuenta este factor. Es evidente que este problema no se presenta con personas que no presenten déficits cognitivos. No es extrapolable a todo el universo de la diversidad funcional. Sin embargo, dado el envejecimiento acelerado de la población y el aumento constante de la expectativa de vida, cada vez es más acuciante desarrollar tecnologías que sean realmente beneficiosas para el ser humano, y no simplemente gadgets o sofisticados juguetes tecnológicos que cumplan su función de forma aséptica olvidándose del respeto debido a nuestros mayores.

\section{Garantías de la privacidad del proceso experimental, de datos personales y productos derivados de la investigación}

Existen diferentes patrones de comportamiento asociados con diferentes relaciones sociales, y estos patrones se establecen de acuerdo a su adecuación a contextos concretos. Por lo tanto, se esperan ciertos patrones de conducta de un profesional en un entorno de trabajo, mientras que un comportamiento diferente se espera de la misma persona en un entorno familiar. En cualquier entorno de investigación, es esencial distinguir entre los diferentes roles que las personas pueden jugar dependiendo de la situación. A veces la privacidad se invade inadvertidamente, y tanto los investigadores como los voluntarios pueden sentir que una parte de su intimidad se pone en juego en esta 
relación específica, ya que es una relación que da derecho a la otra parte para obtener información sobre diferentes aspectos personales. Si no podemos controlar quién tiene acceso a nosotros, y a quién podemos incluir o excluir en nuestras relaciones, puede que no seamos capaces de regular nuestra conducta de forma adecuada. Por ello debe existir un primer nivel de garantías de privacidad que afecte al proceso de investigación como entorno relacional.

Esta importante cuestión, que es especialmente sensible en entornos de colaboración tales como aquellos entornos de diseño participativo, es seguida por otra posición (Swain et al., 1998), en la que los usuarios o los participantes pueden ejercer el control sobre los procesos de recopilación y presentación de datos, y por ello debe haber un sentido de respeto. Recientemente podemos apreciar que se ha dado un aumento en el uso de tecnologías que son omnipresentes e invisibles, tecnologías que no requieren mucho conocimiento por parte del usuario. Estas tecnologías, integradas en el entorno, no permiten a los usuarios elegir su interacción (Mordini et al., 2009). Estos cambios tecnológicos afectarán a la forma en que las personas interactúan con la tecnología y a cómo se desarrollan sus percepciones de estas tecnologías. Los participantes en investigación tecnológica deben ser conscientes de la importancia de evaluar estos acontecimientos y sus consecuencias. Es necesario entender el problema de la privacidad en el ámbito de la investigación de la misma forma que la relación entre, por ejemplo, la privacidad y las tecnologías de la información en el caso de la domótica (Courtney, 2008), especialmente cuando el participante no tiene otra opción. Así que aunque a los participantes se les garantiza, a través de herramientas como el consentimiento informado, evitar cualquier coacción para llevar a cabo una interacción que no desean, debemos tener en cuenta la presión que el medio ambiente puede tener sobre ellos. En el fondo, el conjunto de los datos de carácter personal sensibles para el usuario componen lo que podríamos llamar su cuerpo electrónico. ${ }^{3}$ Este sería un segundo nivel

3. El concepto de cuerpo electrónico (electronic body) puede de garantías relacionadas con la integridad de ese cuerpo electrónico.

Con el fin de garantizar a los participantes que su privacidad es respetada, además de la protección de la información relevante dictada por la legislación sobre protección de datos y la forma en que se tratan estos datos, el formulario de consentimiento informado puede representar e informar acerca de esta garantía. El problema de la información no es solo el robo de la misma, sino el mal uso de la misma por parte de personas del propio entorno del usuario. (Ejemplo: detector de huellas digitales a la puerta de la residencia. Los familiares del usuario pueden saben quién lo visita y por cuanto tiempo, si pasa allí la noche, con qué frecuencia, etc.). Hay que prestar un cuidado especial a la custodia de dicha información, recopilando solo la información que sea necesaria para llevar a cabo una investigación en aras de mejorar la calidad de vida del usuario.

Paralelamente aparece un tercer nivel de garantías, que debe responder al problema de los productos derivados de la investigación y a la extensión subrepticia de la información (function creep): datos de carácter personal que han sido recolectados con un propósito concreto y definido, pueden ser añadidos a otros, mezclados, combinados y extrapolados para otros fines ajenos a la motivación original. Hay datos personales que parecen inocentes, pero una vez combinados son como una bomba de relojería que estalla en nuestras manos, destruyendo cualquier expectativa razonable de privacidad y protección ante la ley. Es necesario prevenir que la empresa que promueve una investigación cambie o extienda el uso de la información personal recogida de los participantes, que sea comprada por otra compañía con otros fines sociales, o vendida a otro país con una regulaciones del derecho de privacidad y control de la información de carácter personal totalmente diferentes. (Bustamante, 20I3).

ser enormemente útil, pues permite aplicar al conjunto de informaciones que provienen del cuerpo de la persona un carácter unitario e identificable. Los principios de la bioética (integridad, dignidad, autonomía del cuerpo, etc.), serían también aplicables a este cuerpo electrónico. 
Nuestra experiencia en la investigación nos ha enseñado que los aspectos éticos relacionados con la investigación que más preocupaba a los participantes eran los relacionados con la privacidad. Este es un resultado esperado (Chen et al., 2002; Müller y Sixsmith, 2008) que nos está diciendo que tenemos que proporcionar información suficiente a los participantes para que puedan desarrollar confianza con los investigadores. Esto es importante no sólo desde el punto de vista de los productos que por medio del diseño participativo se estén desarrollando, sino también en las relaciones que se establecen durante las actividades, y afecta, asimismo, a la confianza que se tiene en las organizaciones implicadas (Nickel, 20II).

\section{Conclusión: Recomendaciones para la investigación con sujetos con diversidad funcional}

Teniendo en cuenta estos datos y nuestra experiencia en el trato con personas con diversidad funcional dentro del ámbito de la investigación tecnológica, podemos recomendar los siguientes pasos cuando se aborda la participación en proyectos de investigación:

- Asegurarse de que el personal investigador o de apoyo es consciente de la necesidad de tener tacto al tratar con personas con diversidad funcional.

- Preparar modelos de consentimientos informados disponibles fáciles de entender para las personas con diversidad cognitiva o déficit de aprendizaje. Las personas con impedimentos visuales también deben tener un formato adecuado para acceder a la información proporcionada en el formulario de consentimiento, así como para registrar su consentimiento de participación en una actividad.

- Promover la expresión de opiniones e intereses por parte de los participantes voluntarios, libremente y en todo momento.

- Desarrollar la confianza de los participantes para que se sientan más cómodos durante las sesiones y estén dispuestos a volver, al tiempo que se evita la estigmatización.

- Ser claro y cuidadoso en la presentación y uso de tecnologías para evitar posibles sentimientos de decepción al terminar la investigación.

- Garantizar la privacidad en los procedimientos experimentales y asegurar que la información de carácter personal recopilada para la investigación o generada en el curso de la misma no es utilizada para otros fines.

- Debe haber una información clara y consistente, presentada de forma adecuada a la TE, acerca de los fines, los procedimientos y la metodología del proyecto. Aplicable al consentimiento informado y las instrucciones de uso de cada tecnología.

- Habrá que definir la responsabilidad de cada uno de los actores. Cada parte del sistema debe tener una parcela de responsabilidad definida e identificada, con claras fronteras con respecto a las demás. Desde el punto de vista legal, no hay ningún problema, ya que las responsabilidades se delimitan por contrato. Desde el punto de vista ético, es conveniente profundizar en esta noción. 
Referencias bibliográficas

Barcenilla, J. y Tijus, C. (2OI2): "Ethical issues raised by the new orientations in ergonomics and living labs", Work: A Journal of Prevention, Assessment and Rehabilitation, 4I (o):5259-5265. IOS Press.

Bustamante, J. (2013): “Ética en la nube: dilemas éticos y políticos en el modelo de Computación en nube (Ethics on the cloud: ethical and political dilemmas in the model of Cloud computing)", en Argumentos de Razón Técnica, 16: 37-54.

Chen, L., Gillenson, M. L., y Sherrell, D. L. (2002): "Enticing online consumers: an extended technology acceptance perspective", Information and Management, 39: 705-7I9.

Courtney, K. L. (2008): "Privacy and senior willingness to adopt smart home information technology in residential care facilities", Methods Inf Med, 47(I):, 76-8I.

Gram-Hansen, S. B. (2009): “Towards an approach to ethics and HCI development based on Løgstrup's ideas”, In Gross, T. et al. (eds.): Interact 2009, Part I, Springer Verlag, LNCS 5726: 200-203.

Manders-Huits, N. (2010): "What values in design? The challenge of incorporating moral values into design", Science and Engineering Ethics, I7: 271-328.

Mordini, E. et al. (2009): "Senior citizens and the ethics of e-inclusion", Ethics and Information Technology, I I: 203-220.

Müller, S. y Sixsmith, A. (2008): “User requirements for ambient assisted living: results of the SOPRANO project”, Gerontechnology, 7 (2): I 68.

Nys, H. (20I2): “New European Rules Regarding the Approval of Clinical Trials, the Role of Ethics Committees and the Protection of Subjects", Archivum Immunologiae et Therapiae Experimentalis, 60 (6): 405-4I 4 .

Nickel, P. J., (20I I): "Ethics in e-trust and e-trustworthiness : the case of direct computerpatient interfaces", Ethics and Information Technology, I3 (4): 355-363.

Stalker, K. (I998): “Some Ethical and Methodological Issues in Research with People with Learning Difficulties”, Disability and Society, I3 (I): 5-I9.

Swain, J., Heyman, B. y Gillman, M. (I998): "Public Research, Private Concerns: Ethical issues in the use of open-ended interviews with people who have learning difficulties", Disability and Society, I 3 (I): 2I-36.

Thiel, C. E. et al. (2012): "Leader Ethical DecisionMaking in Organizations: Strategies for Sensemaking", Journal of Business Ethics, IO7 (I): 49-64.

Turilli, M. (2007): "Ethical protocol design", Ethics and Information Technology, 9: 49-62.

Vines J. et al. (2012): "Why involve people in design?" In: ACM Conference on Human Factors in Computing Extended Abstracts (CHI EA 'I 2) 20I 2, Austin, Texas: ACM Press. 